\title{
Existence, uniqueness and other properties of the BCT (minimal strain lapse and shift) gauge
}

\author{
David Garfinkle \\ Department of Physics, Oakland University, Rochester, MI 48309, USA \\ Carsten Gundlach \\ Faculty of Mathematical Studies, University of Southampton, Southampton SO17 1BJ, United Kingdom \\ James Isenberg \\ Department of Mathematics, University of Oregon, Eugene, OR 97403, USA \\ Niall ÓMurchadha \\ Physics Department, University College, Cork, Ireland
}

(31.3.00)

\begin{abstract}
Brady, Creighton and Thorne have proposed a choice of the lapse and shift for numerical evolutions in general relativity that extremizes a measure of the rate of change of the three-metric (BCT gauge). We investigate existence and uniqueness of this gauge, and comment on its use in numerical time evolutions.
\end{abstract}

\section{THE BCT GAUGE}

Cauchy data for general relativity consist of a three-metric $g_{a b}$ and extrinsic curvature $K_{a b}$ specified on a threemanifold ("slice") $\Sigma$. The Cauchy data determine the four-dimensional spacetime (locally) as a geometric object, but without fixing a coordinate system. When the spacetime is computed numerically as a sequence of spacelike slices $\Sigma(t)$, the coordinates may be fixed incrementally by specifying a lapse $\alpha$ and shift vector $\beta^{a}$ on each new slice - a gauge choice. In one class of gauge choices, $\alpha$ and $\beta^{a}$ on a slice are determined by $g_{a b}$ and $K_{a b}$ on the same slice, i.e.,

$$
\left(\Sigma, g_{a b}, K_{a b}\right) \rightarrow\left(\alpha, \beta^{a}\right)
$$

Recently, Brady, Creighton and Thorne [1] have proposed a new gauge choice of this class in which $\alpha$ and $\beta^{a}$ are determined as a solution of the coupled equations

$$
K^{a b} F_{a b}=0, \quad D^{a} F_{a b}=0,
$$

where $D_{a}$ is the covariant derivative with respect to the three-metric $g_{a b}$, and

$$
F_{a b}(\alpha, \beta) \equiv L_{a b}(\beta)-2 \alpha K_{a b}, \quad L_{a b}(\beta) \equiv D_{a} \beta_{b}+D_{b} \beta_{a}
$$

These two equations arise when one varies the action principle

$$
I=\int F^{a b} F_{a b} \sqrt{g} d^{3} x
$$

with respect to $\alpha$ and $\beta^{b}$, respectively. In a four-dimensional context, $F_{a b}$ is the time derivative of the three-metric induced on the time slicing with lapse $\alpha$ and shift $\beta^{a}$ :

$$
\dot{g}_{a b} \equiv \mathcal{L}_{t} g_{a b}=F_{a b}(\alpha, \beta), \quad t^{a} \equiv \alpha n^{a}+\beta^{a},
$$

where $n^{a}$ is the unit normal vector of the slice $\Sigma$ when it is embedded into a spacetime. Equations (2) are therefore also called the minimal strain lapse equation and minimal strain shift equation, where $I$ is the "strain" that is being extremized.

The motivation for considering these equations is that a good gauge choice should have the property (among others) to be compatible with approximate Killing vectors, in the sense that if an approximate Killing vector exists, the spacetime metric in that gauge should evolve as slowly as possible. The inspiral phase of a binary black hole system in a spherical orbit, for example, has an approximate Killing vector, and one would like to be able to find (corotating) coordinates in which the spacetime metric evolves on the timescale in which the orbit shrinks through the emission of gravitational waves, rather than on the much shorter orbital period timescale. 
The first of the two equations (2) can be solved algebraically for $\alpha$,

$$
\alpha=\frac{K^{c d} L_{c d}(\beta)}{K^{e f} K_{e f}},
$$

and the result substituted into the second equation. One obtains a vector linear second-order differential equation for the vector $\beta$ alone:

$$
D^{a} H_{a b}(\beta)=0, \quad H_{a b}(\beta) \equiv L_{a b}(\beta)-2 \frac{K^{c d} L_{c d}(\beta)}{K^{e f} K_{e f}} K_{a b}
$$

We shall call this single vector equation the BCT equation, and in the following we shall consider the lapse $\alpha$ as a dependent quantity determined by (6). Equation (7) can be obtained from the action principle

$$
J=\int H^{a b} H_{a b} \sqrt{g} d^{3} x,
$$

which is obtained by substituting the lapse (6) into the action principle $I$.

\section{EXISTENCE AND UNIQUENESS}

If one is to use the BCT gauge, it is important to know the answer to the following question: For which choices of data $\left(U, g_{a b}, K_{a b}\right)$ on a region $U$ of $\Sigma$ with boundary $\partial U$ and for which sets of boundary conditions for $\beta$ can one solve the BCT equation (7)? In this brief note, we show that for some choices of data there is a unique solution with specified Dirichlet boundary conditions, while for others, there are many solutions with those boundary conditions.

The issues of existence and uniqueness of the BCT gauge have been previously considered by Gonçalves [2. Gonçalves shows that the differential operator defined by (7) is strongly elliptic if and only if $K_{a}{ }^{b}$, considered as a map on the tangent space of $\Sigma$, has at most one vanishing eigenvalue. (Let the principal part of the differential operator acting on $\beta^{a}$ be $M_{b}^{a}{ }_{b}^{c d} D_{c} D_{d} \beta^{b}$. The operator is then defined to be strongly elliptic if $M_{b}^{a}{ }^{c d}$ is positive definite with respect both to the two indices that slot into derivatives and the index pair that shuffles the vector index on $\beta$.) Generally, operator ellipticity - strong or otherwise - is not enough to determine whether a boundary value problem admits a solution. However, using the fact that the equation of interest (7) is of divergence form, Gonçalves does obtain local results. In this paper, using the Fredholm alternative, we obtain stronger, global, results.

Before deriving the condition for existence of the BCT gauge, we wish to turn the boundary value problem for Equation (7) into one with homogeneous boundary conditions. Let us write Equation (7) in operator form as $O(\beta)=0$, and the corresponding Dirichlet problem as

$$
O(\beta)=0,\left.\quad \beta\right|_{\partial U}=f
$$

for some given continuous vector-valued function $f$. Presuming that the region $U$ and its boundary are well-behaved, we may always extend $f$ to a function $F: U \rightarrow R^{3}$ which is $C^{2}$ on $U$ and continuous on $U \cup \partial U$. Then if we find a solution $\xi$ of the boundary value problem

$$
O(\xi)=-O(F),\left.\quad \xi\right|_{\partial U}=0,
$$

and if we set $\beta=\xi+F$, we have $\beta$ satisfying the boundary value problem (9). We may now focus on the discussion of the boundary value problem (10) (for arbitrary $F$ ).

To be able to use Fredholm ideas to study the boundary value problem (10), one first needs to establish that (10) defines a Fredholm map. As shown in propositions 11.10 and 11.16 of [3], this fact follows from the strong ellipticity of $O$. We also need the following:

Lemma: For $\beta$ satisfying the Dirichlet condition $\left.\beta\right|_{\partial U}=0, O$ is a self-adjoint operator.

Proof: We consider the quantity

$$
\int_{U} \gamma O(\beta) \equiv \int_{U} \gamma^{a} D^{b} H_{a b}(\beta)
$$

with $H_{a b}$ from Equation (7). Integrating by parts, and using $\left.\gamma\right|_{\partial U}=0$, we obtain 


$$
\int_{U} \gamma O(\beta)=-\int_{U} D^{b} \gamma^{a} H_{a b}(\beta)=-\int_{U} H^{a b}(\gamma) D_{a} \beta_{b}
$$

Integrating by parts again, and using $\left.\beta\right|_{\partial U}=0$, we have

$$
\int_{U} \gamma O(\beta)=\int_{U} D_{a} H^{a b}(\gamma) \beta_{b}=\int_{U} \beta O(\gamma)
$$

Hence $O$ is self-adjoint on functions $\beta$ that vanish on the boundary. (As the boundary term in the integration by parts is of the form $\int_{\partial U} s^{a} \beta^{b} H_{a b}(\gamma)$, where $s^{a}$ is the unit normal vector to $\partial U$, the operator $O$ is also self-adjoint on the space of functions $\gamma$ that satisfy $s^{a} H_{a b}(\gamma)=0$ on the boundary. This boundary condition is the mathematical analog of the Neumann boundary condition for the Laplace equation, but it contains additional terms that make its physical meaning unclear. Therefore we do not consider it here.)

Since $O$ is self-adjoint, and since it defines a Fredholm map, the Fredholm Alternative [3] specifies a clear condition for existence of solutions. Stated for the operator $O$, one has

Proposition (Fredholm Alternative): Fix $\left(U, g_{a b}, K_{a b}\right)$. The Dirichlet boundary value problem (10) has a unique solution for every choice of $O(F)$ if and only if the only vector function satisfying

$$
O(\xi)=0,\left.\quad \xi\right|_{\partial U}=0
$$

is $\xi^{a}=0$. (That is, the kernel of $O$ is empty.) For a given $O(F)$, the boundary value problem (10) has a solution so long as $O(F)$ satisfies

$$
\int_{U} \xi O(F)=0
$$

for every $\xi$ which satisfies (14). (The kernel of $O$ is orthogonal to the source.)

We base our existence results for the BCT gauge on the Fredholm Alternative. So we are led to consider solutions $\xi$ of (14), or elements of the kernel of $O$. Given such a solution, we consider the quantity

$$
\int_{U} \xi O(\xi)=-\frac{1}{2} \int_{U} L^{a b}(\xi) H_{a b}(\xi)=-\frac{1}{2} \int_{U} H^{a b}(\xi) H_{a b}(\xi),
$$

where the first equality follows from integration by parts ( $\xi$ vanishes on $\partial U$ ) and the second equality follows from $K^{a b} H_{a b}=0 . \xi$ therefore satisfies (14) if and only if

$$
H_{a b}(\xi)=0,\left.\quad \xi\right|_{\partial U}=0 .
$$

Thus the kernel of $O$ consists of solutions of this boundary value problem.

For a given set of initial data $\left(U, g_{a b}, K_{a b}\right)$, the Fredholm Alternative asks that we determine the kernel of $O$. If the kernel is empty, then the Dirichlet boundary value problem (10), and hence (9), admits a unique solution.

If the kernel is not empty, with non-trivial elements $\xi$, then to determine the solubility of the boundary value problem (10) we need to consider $\int_{U} \xi O(F)$. Integrating by parts, we have

$$
\int_{U} \xi O(F)=-\frac{1}{2} \int_{U} L(\xi) H(F)=-\frac{1}{2} \int_{U} H(\xi) L(F),
$$

but $H(\xi)=0$ by assumption. So this always vanishes, and a solution always exists in this case, too. It is determined, however, only up to the addition of any element of the kernel.

We conclude that a solution of the BCT gauge exists whenever $O$ is strongly elliptic, but if the kernel (17) is not empty, the solution is not unique.

\section{EXAMPLES FOR UNIQUENESS AND NON-UNIQUENESS}

It is useful to note that there are choices of data $\left(U, g_{a b}, K_{a b}\right)$ for which each of these two cases hold. For the first case, where the kernel is empty, we consider data with $K_{a b}=\rho g_{a b}$, with $\rho$ nowhere vanishing. The kernel equation (17) then becomes

$$
D_{a} \xi_{b}+D_{b} \xi_{a}-\frac{2}{3} g_{a b} D^{c} \xi_{c}=0
$$


This is the equation for a conformal Killing vector. If we choose a metric $g_{a b}$ that does not admit a conformal Killing vector that vanishes on the boundary, we have constructed data that give rise to an empty kernel, and therefore a unique BCT gauge for a given choice of the boundary data.

For the other case, we consider a slice through a spacetime that has a timelike Killing vector. We choose the lapse and shift so that $t^{a}$ is the Killing vector. The time derivative of the three-metric then vanishes, and consequently $F_{a b}(\alpha, \beta)=0$. From this it follows that $H_{a b}(\beta)=0$. In order to obtain $\left.\beta\right|_{\partial U}=0$, we choose the slice so that it is normal to the Killing vector in $\partial B$ but not in $B$. If in such a situation one tries to solve the BCT equation with a boundary where the slice is approximately normal to the Killing vector, the numerical problem might become badly conditioned.

As a concrete example, we consider a spherically symmetric slice in flat spacetime. Let $(t, r, \theta, \varphi)$ be the standard spherically coordinates on Minkowski spacetime, and let the slice be given by $t=T(r)$. As coordinates intrinsic to the slice we use $(r, \theta, \varphi)$ induced by the spacetime coordinates of the same name. The normal vector to the slice, induced 3-metric and extrinsic curvature are then given by

$$
\begin{aligned}
n^{r} & =\frac{T^{\prime}}{\sqrt{1-T^{\prime 2}}}, \quad n^{t}=\frac{1}{\sqrt{1-T^{\prime 2}}}, \\
g_{r r} & =1-T^{\prime 2}, \quad g_{\theta \theta}=r^{2}, \quad g_{\varphi \varphi}=r^{2} \sin ^{2} \theta, \\
K_{r r} & =-\frac{T^{\prime \prime}}{\sqrt{1-T^{\prime 2}}}, \quad K_{\theta \theta}=-\frac{r T^{\prime}}{\sqrt{1-T^{\prime 2}}}, \quad K_{\varphi \varphi}=\sin ^{2} \theta K_{\theta \theta} .
\end{aligned}
$$

The normal vector is parallel to the Killing vector $\partial / \partial t$ where $T^{\prime}(r)=0$, say at $r=r_{0}$. We choose $U$ to be the ball $r \leq r_{0}$. The desired element of the kernel of $O$ is then

$$
\alpha=\sqrt{1-T^{\prime 2}}, \quad \beta^{r}=-T^{\prime},
$$

up to an overall constant factor.

The potential difficulty with non-uniqueness can be avoided by an appropriate choice of slice and boundary. In particular, for the choice of slice and boundary proposed in [1] for the binary black hole problem, the shift is nowhere small, so that the Killing vector is nowhere normal to the slice. Near the black hole excision boundary, the slicing is of the Painlevé-Güllstrand (or Kerr-Schild) type, with a large radial shift, while at the outer boundary the coordinates are corotating, with a large $\partial / \partial \phi$ shift component.

\section{USE OF THE BCT GAUGE AND OTHER GAUGES IN TIME EVOLUTION}

Consider the evolution of initial data $\left(g_{a b}, K_{a b}\right)$. The evolution equation for $K_{a b}$ is of the form

$$
\dot{K}_{a b}=-D_{a} D_{b} \alpha+\text { other terms. }
$$

The time derivative of $K_{a b}$ contains the second spatial derivative of the lapse. If one uses the BCT gauge to determine $\alpha$ and $\beta$ from $g_{a b}$ and $K_{a b}$, the evolution equation for $K_{a b}$ becomes

$$
\dot{K}_{a b}=-\left[\frac{L^{c d}(\beta)}{K_{e f} K^{e f}}-2 \frac{L^{m n}(\beta) K_{m n} K^{c d}}{\left(K_{e f} K^{e f}\right)^{2}}\right] D_{a} D_{b} K_{c d}+\text { other terms. }
$$

This means that if $K_{a b}$ is initially in a function space of finite differentiability, time evolution takes it out of that space - it "loses two derivatives". ( $\beta^{a}$ itself appears to gain two derivatives because it is the solution of an elliptic equation, but this does not affect the argument.)

While this is a technical obstacle to proving existence and uniqueness of solutions to the Einstein equations in the $\mathrm{BCT}$ gauge, it also hints at the possible existence of a practical problem for the use of the BCT gauge in numerical evolution. Roughly speaking, one would expect numerical noise to be amplified during time evolution, whereas maximal slicing with zero shift, for example, is empirically known to dampen numerical noise. In the toy model equation $\dot{u}=\kappa u_{x x}$, noise is damped for $\kappa>0$ (heat equation) and increases for $\kappa<0$ (heat equation run backwards), so that one only needs to choose the correct sign of $\kappa$. In contrast, Equation (25) is a nonlinear tensor equation. It appears plausible that some of the eigenvalues of its linearization around some backgrounds $\left(g_{a b}, K_{a b}\right)$ correspond to negative $\kappa$, thus leading to the growth of linear noise. Corresponding nonlinear instabilities may also exist. We have not attempted to investigate this question. 
Here we would like to draw attention to an alternative gauge choice. Consider the following coupled equations for the lapse and shift:

$$
\begin{aligned}
D_{b} D^{b} \beta^{a}+D_{b} D^{a} \beta^{b}-2 D_{b}\left(\alpha K^{a b}\right)-\frac{n}{3} D^{a}\left(2 D_{b} \beta^{b}-2 \alpha K\right) & =0, \\
-D_{a} D^{a} \alpha+\left[{ }^{(3)} R+K^{2}+\frac{1}{2}(\tau-3 \rho)\right] \alpha+\beta^{a} D_{a} K & =0 .
\end{aligned}
$$

Here $\tau$ and $\rho$ are matter terms, namely the trace of the (three-dimensional) stress tensor and the energy density. $n$ is a constant that is either zero or one. These equations for $\alpha$ and $\beta$ are elliptic, but they are not self-adjoint. Existence and uniqueness of solutions will be considered elsewhere [4]. As the equations are elliptic in both $\alpha$ and $\beta,\left(\dot{g}_{a b}, \dot{K}_{a b}\right)$ do not lose differentiability compared to the Cauchy data $\left(g_{a b}, K_{a b}\right)$.

The first, vector, equation is $D_{b} F^{a b}=0$ for $n=0$, and is $D_{b}\left(F^{a b}-1 / 3 g^{a b} F_{c}^{c}\right)=0$ for $n=1$. These two shift conditions were suggested by Smarr and York in their classic paper on coordinate conditions [5] under the names "minimal strain shift" and "minimal distortion shift". The second, scalar, equation is $\dot{K}=0$. For $K=0$ it reduces to maximal slicing. Here, however, we do not assume that $K$ is zero, nor that it is constant in space. $\dot{K}=0$ slicing, and its combination with minimal distortion shift, was suggested by Smarr and York, and they also discuss its Killing vector-tracking property. Their subsequent discussion focuses on maximal $(K=0)$ slicing with minimal distortion shift. This more restricted gauge choice is now commonly associated with the name "Smarr-York" (SY) gauge, while the more general gauge with $K \neq 0$ seems to have been forgotten. The desirability of Killing vector tracking was later rediscovered in [1] and [6], and the gauge discussed here was rediscovered as "generalized Smarr-York" (GSY) gauge in [6].

Here we want to point out that the GSY gauge has the desirable properties of the BCT gauge - it tracks Killing vectors and it admits generic Cauchy data - without having the "loss of derivatives" property and the possible problem when the slice is normal to the Killing vector at the boundary. In a direct numerical comparison of the BCT and GSY gauges in spherical symmetry, Garfinkle and Gundlach [6] find that the GSY gauge stably tracks Killing vectors, but are unable to obtain a stable time evolution with the BCT gauge, and this may be due to the "loss of derivatives" property. Shibata [7] reports good experiences with an approximate implementation of maximal slicing with minimal strain shift in 3D evolutions of a neutron star binary. Independent numerical work, and tests in 3D, are required to decide if one of the gauges is more suitable in practice than the other.

\section{ACKNOWLEDGMENTS}

This work was carried out during a mini-program on "Colliding Black Holes" at the Institute for Theoretical Physics, Santa Barbara, where it was supported by NSF grant PHY-9407194. DG is also supported NSF grant PHY-9722039 to Oakland University, and JI by grant PHY-9800732 to the University of Oregon.

[1] P. R. Brady, J. D. Creighton and K. S. Thorne, Phys. Rev. D 58, 061501 (1998).

[2] S. M. C. V. Gonçalves, Integrability of the minimal strain equations for the lapse and shift in $3+1$ numerical relativity, e-print gr-qc/9911020v2.

[3] M. E. Taylor, Partial Differential Equations (vol. 1), Springer, 1996.

[4] N. ÓMurchadha, in preparation.

[5] L. Smarr and J. W. York, Jr., Phys. Rev. D 17, 2529 (1978).

[6] D. Garfinkle and C. Gundlach, Class. Quant. Grav. 16, 4111 (1999).

[7] M. Shibata, Phys. Rev. D 60, 104052 (1999). 